\title{
Nanoplasma Dynamics of Single Large Xenon Clusters Irradiated with Superintense X-Ray Pulses from the Linac Coherent Light Source Free-Electron Laser
}

T. Gorkhover, ${ }^{1}$ M. Adolph, ${ }^{1}$ D. Rupp, ${ }^{1}$ S. Schorb,,${ }^{1,2}$ S. W. Epp,${ }^{3,4}$ B. Erk,,${ }^{3,4}$ L. Foucar,${ }^{3,5}$ R. Hartmann, ${ }^{6}$ N. Kimmel, ${ }^{7,8}$ K.-U. Kühnel, ${ }^{4}$ D. Rolles, ${ }^{3,5}$ B. Rudek,${ }^{3,4}$ A. Rudenko, ${ }^{3,4}$ R. Andritschke, ${ }^{7,8}$ A. Aquila, ${ }^{9,10}$ J. D. Bozek, ${ }^{2}$ N. Coppola, ${ }^{9,10}$ T. Erke, ${ }^{9}$ F. Filsinger,${ }^{11}$ H. Gorke, ${ }^{12}$ H. Graafsma, ${ }^{9}$ L. Gumprecht, ${ }^{9}$ G. Hauser,${ }^{7,8}$ S. Herrmann,${ }^{7,8}$ H. Hirsemann, ${ }^{9}$ A. Hömke, ${ }^{3,4}$ P. Holl, ${ }^{6}$ C. Kaiser, ${ }^{4}$ F. Krasniqi, ${ }^{3,5}$ J.-H. Meyer, ${ }^{9}$ M. Matysek, ${ }^{13}$ M. Messerschmidt,${ }^{2}$ D. Miessner, ${ }^{7,8}$ B. Nilsson, ${ }^{9}$ D. Pietschner, ${ }^{7,8}$ G. Potdevin, ${ }^{9}$ C. Reich, ${ }^{6}$ G. Schaller, ${ }^{7,8}$ C. Schmidt, ${ }^{3,4}$ F. Schopper, ${ }^{7,8}$ C. D. Schröter, ${ }^{4}$ J. Schulz, ${ }^{9,10}$ H. Soltau ${ }^{6}$ G. Weidenspointner, ${ }^{7,8}$ I. Schlichting, ${ }^{5,3}$ L. Strüder, ${ }^{3,7,8,14}$ J. Ullrich, ${ }^{3,4,15}$ T. Möller, ${ }^{1}$ and C. Bostedt ${ }^{2, *}$

${ }^{1}$ Institut für Optik und Atomare Physik, Technische Universität Berlin, Hardenbergstr. 36, 10623 Berlin, Germany

${ }^{2}$ Linac Coherent Light Source, SLAC National Accelerator Laboratory, Stanford, California 94309, USA

${ }^{3}$ Max Planck Advanced Study Group, Center for Free-Electron Laser Science, Notkestrasse 85, 22607 Hamburg, Germany

${ }^{4}$ Max-Planck-Institut für Kernphysik, Saupfercheckweg 1, 69117 Heidelberg, Germany

${ }^{5}$ Max-Planck-Institut für medizinische Forschung, Jahnstr. 29, 69120 Heidelberg, Germany

${ }^{6}$ PNSensor GmbH, Otto-Hahn-Ring 6, 81739 München, Germany

${ }^{7}$ Max-Planck-Institut Halbleiterlabor, Otto-Hahn-Ring 6, 81739 München, Germany

${ }^{8}$ Max-Planck-Institut für extraterrestrische Physik, Giessenbachstrasse, 85741 Garching, Germany

${ }^{9}$ Photon Science, DESY, Notkestrasse 85, 22607 Hamburg, Germany

${ }^{10}$ European XFEL GmbH, Albert-Einstein-Ring 19, 22761 Hamburg, Germany

${ }^{11}$ Fritz-Haber-Institut der Max-Planck Gesellschaft, Faradayweg 4-6, 14195 Berlin, Germany

${ }^{12}$ Forschungszentrum Jülich, Institut ZEL, 52425 Jülich, Germany

${ }^{13}$ Inst. f. Experimentalphysik, Universität Hamburg, 22607 Hamburg, Germany

${ }^{14}$ Universität Siegen, Emmy-Noether Campus, Walter Flex Str. 3, 57068 Siegen, Germany

${ }^{15}$ Physikalisch-Technische Bundesanstalt (PTB), Bundesallee 100, 38116 Braunschweig, Germany

(Received 13 February 2012; published 15 June 2012)

The plasma dynamics of single mesoscopic Xe particles irradiated with intense femtosecond x-ray pulses exceeding $10^{16} \mathrm{~W} / \mathrm{cm}^{2}$ from the Linac Coherent Light Source free-electron laser are investigated. Simultaneous recording of diffraction patterns and ion spectra allows eliminating the influence of the laser focal volume intensity and particle size distribution. The data show that for clusters illuminated with intense x-ray pulses, highly charged ionization fragments in a narrow distribution are created and that the nanoplasma recombination is efficiently suppressed.

Free-electron lasers (FELs) such as the Linac Coherent Light Source (LCLS) deliver extremely intense and coherent $\mathrm{x}$-ray flashes with a femtosecond pulse length, opening the door for imaging single nanometer-sized objects with atomic resolution in single shots [1,2]. All matter irradiated by the intense $\mathrm{x}$-ray pulses will be highly ionized within femtoseconds. So far, investigations about the ionization dynamics under such conditions have focused on ensembles of atoms and small systems on the one hand [3-6] and macroscopic solids on the other hand [7]. A detailed understanding of the $\mathrm{x}$-ray pulse-matter interaction for intermediate, i.e., mesoscopic, systems is of utmost relevance for x-ray imaging applications. Most samples to be imaged with intense $\mathrm{x}$-ray laser pulses will be in the size regime of a few tens to hundreds of nanometers and first results on their pulse-length dependent scattering response are controversial $[8,9]$.

For studying fundamental questions of the light-matter interaction, atomic rare gas clusters have proven to be ideal targets $[10,11]$. They are intermediate between atoms and bulk solids, and energy dissipation into surrounding media is virtually absent due to their finite size. First results from short-wavelength FELs have shown that even in the vacuum-ultraviolet spectral regime, the clusters can still be very efficiently heated with inverse bremsstrahlung (IBS) [12]. For shorter wavelength, IBS becomes negligible and the ionization starts being dominated by multistep photoionization $[13,14]$. In the intense $\mathrm{x}$-ray focus of the LCLS with fluences exceeding $10^{5}$ photons $/ \AA^{2}$ atoms are sequentially ionized from the inside out starting with the inner-shell electrons followed by subsequent innershell vacancy decay [3]. In clusters, after a certain number of ionization events, the particle is charged to a degree that its Coulomb energy becomes higher than the kinetic energy of the sequentially ejected electrons and a nanoplasma is formed. In dense nanoplasma, the cluster ionization can be further enhanced by energy-exchanging electron collisions [15]. For photon energies of $\approx 100 \mathrm{eV}$, large clusters are 
transiently highly charged [16] before they disintegrate in a hydrodynamic expansion accompanied by recombination of the cluster core [17-19].

In this Letter we present pioneering results on the ionization and recombination dynamics of single nanometersized samples in intense $x$-ray pulses from LCLS. We have used a novel coincident imaging and spectroscopy approach for the investigation of individual clusters in single shots. Analysis of the scattering patterns allows downselection of the data to events with only a single particle with a defined size in the $\mathrm{x}$-ray focus, therefore providing a unique insight into the intense $\mathrm{x}$-ray pulse-matter interaction. Being able to determine the exposure intensity and size for every single particle, we find drastically different plasma dynamics for different power densities which are not observable in experiments averaging over the focal volume and cluster size distribution [5]. At the highest intensities in the center of the focus, fragments from a highly charged nanoplasma are detected with an unexpected absence of low charge states indicating that plasma recombination during the cluster expansion is efficiently suppressed. The results show that for the high peak-power and photon energies available at LCLS, the ionization and disintegration dynamics of nanoscale samples differ substantially from those found in earlier experiments in the soft $x$-ray regime.

The experiments were performed in the CAMP end station [20] at the AMO beam line of LCLS [21]. The FEL pulses with a photon energy of $800 \mathrm{eV}$ and average pulse energies of $1.5 \mathrm{~mJ}$ were focused to a spot size of about 6 micron (FWHM) diameter. The electron bunch length was set to $200 \mathrm{fs}$ and the $\mathrm{x}$-ray pulse length has been measured to be about $2 / 3$ of that value [22], yielding

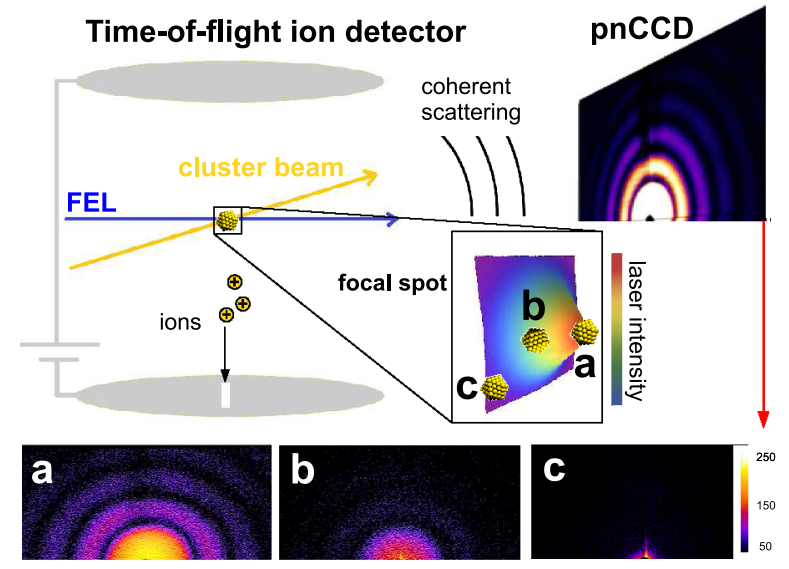

FIG. 1 (color online). Experimental setup (top panel) and single-shot scattering images from individual clusters (bottom panel). The cluster beam is crossed with the focused x-ray beam above the aperture of a time-of-flight spectrometer. The scattered photons are detected by pnCCDs covering scattering angles from $0.5^{\circ}$ to $5^{\circ}$. The scattering signal exhibits a strong intensity variation due to the different positions (a)-(c) of the clusters in the focus as depicted in the inset. an estimated $\mathrm{x}$-ray pulse length of $130 \mathrm{fs}$ for the current experiment. With a beam line transmission on the order of $20 \%$, the resulting power densities exceed $10^{16} \mathrm{~W} / \mathrm{cm}^{2}$. Clusters with average radii of $30 \mathrm{~nm}$ were formed by supersonic expansion of xenon at 9 bar backing pressure through a 200 -micron conical nozzle with a half-opening angle of $4^{\circ}$ cooled to $245 \mathrm{~K}$. The pulsed cluster jet was skimmed twice and a piezo-driven slit skimmer right before the interaction region was adjusted such that, on average, less than one particle was in the FEL focus as evidenced by the single-shot scattering patterns. The cluster beam was crossed with the focussed $\mathrm{x}$-ray beam above the aperture of an ion time-of-flight spectrometer. Conically shaped spectrometer electrodes allowed for a free line of sight from the interaction region to the photon detector. The scattered photons were detected by single photon counting pnCCDs covering scattering angles from $0.5^{\circ}-5^{\circ}[20]$. All data were stored on a shot-to-shot basis and correlated to the FEL parameters.

The experimental setup as well as three representative single-cluster scattering patterns are shown in Fig. 1. The images are used to fit the cluster sizes and filter the data such that only hits from single clusters with similar radii of

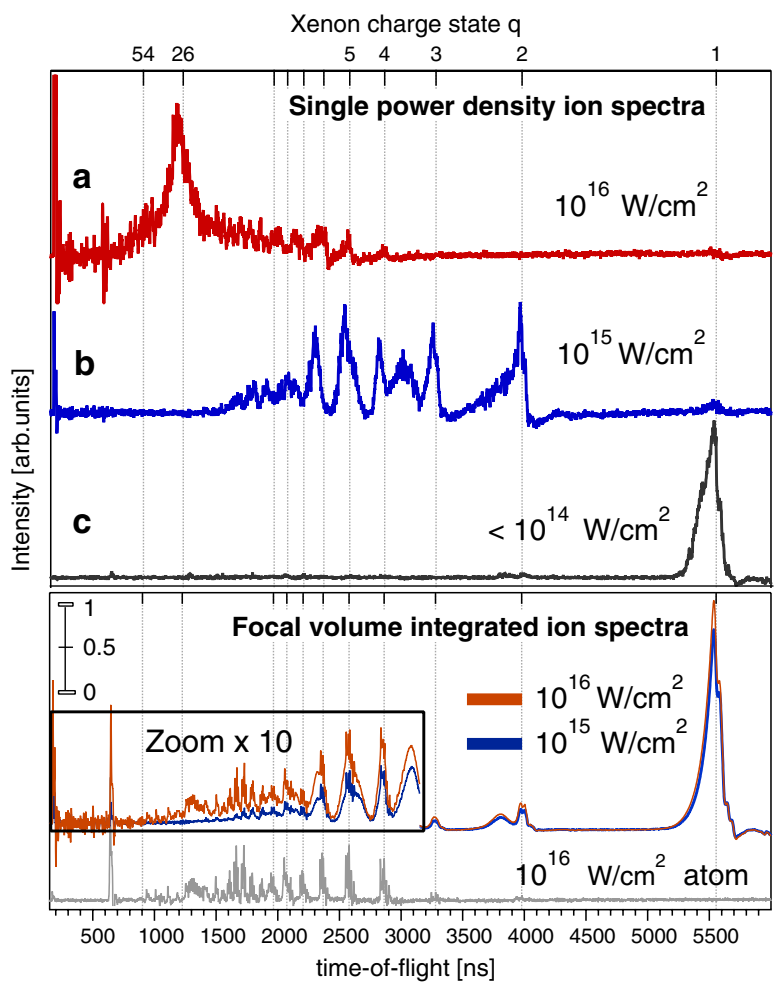

FIG. 2 (color online). Top panel: Single shot ion spectra recorded in coincidence with the scattering images (a)-(c) shown in Fig. 1. Bottom panel: Focal volume integrated ion yield spectra for cluster (top) and atomic (bottom) targets. The single-shot spectra strongly correlate with the position of the cluster in the FEL focus and are strikingly different from the integrated data. 
$30 \pm 2 \mathrm{~nm}$ are used for further analysis, effectively eliminating the influence of the particle size distribution common to experiments with supersonic expansion sources. The total number of scattered photons for individual images is proportional to the FEL power density experienced by the respective single clusters. The scattered photon intensity varies strongly from shot to shot [cf. bottom panels (a)-(c) in Fig. 1] even for clusters of similar size due to the fluctuations of their position in the FEL focus waist. The integrated scattering signal, i.e., the impinging photon intensity experienced by the cluster declines by about one order of magnitude between cases (a), (b), and (c), respectively.

The ion yield spectra from single clusters recorded in coincidence with the scattering patterns from Fig. 1 are shown in the top panel of Fig. 2. Additionally, in the bottom panel of Fig. 2, focal-volume integrated spectra are presented for two different power densities as well as an atom reference spectrum. The three single-cluster spectra reveal strong power-density dependencies. For the best-hit case (a) the ion yield is centered around a charge state of $q=26^{+}$and $q<4^{+}$are completely absent. At the same time, $26^{+}$is also the high-charge state cutoff in the atomic reference recorded at a similar peak power density (bottom panel Fig. 2) which will be discussed further below. The broadening of the peak in the cluster spectrum is attributed to the kinetic energy release of the cluster fragments, but the existence of charge states beyond the atomic ionization limit cannot be excluded from the current measurement. For the cluster illuminated with about a factor of ten less power density [case (b)], the charge distribution shifts to significantly lower $q$. Proceeding to a factor 100 less power density [case (c)], only singly-charged fragments are left, very similar to experiments in the single x-ray photon limit [23]. The single-cluster data are in striking contrast to the focal volume integrated results in the lower panel of Fig. 2. Here, the spectra are dominated by singly-charged fragments and low charge states in agreement with an earlier study [5]. Decreasing the power density by an order of magnitude, similar to the conditions of the single-particle experiments described above, leads to a significant suppression of the high charge states while the intensity of low $q$ remains rather similar.

The coincident imaging and spectroscopy data from single clusters demonstrate the existence of strong power-density-dependent dynamical processes that are washed out in the integrated data. This becomes particularly obvious for the highest power densities, where low charge states are entirely absent, a feature that has never been observed in nonlinear light-matter interaction experiments in other spectral regimes $[10,11,24]$. To shed more light on the transition from the low to the high power density spectra, the dominant charge state as well as the average charge state from single clusters with similar radii $(30 \pm 2 \mathrm{~nm})$ are compared in Fig. 3. The average charge

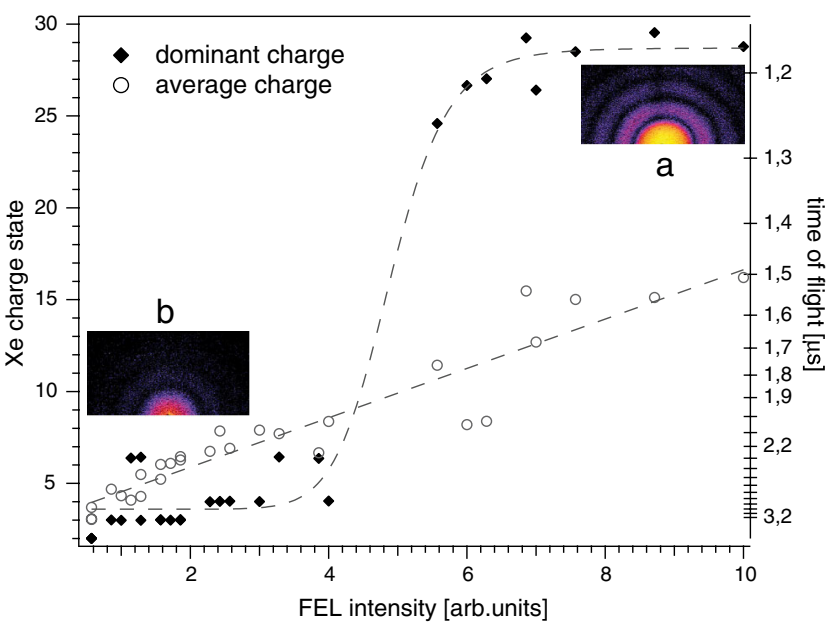

FIG. 3 (color online). Average (open circles) and the dominant charge state (filled diamonds) for increasing FEL intensities. The FEL intensities are deduced from the integrated scattering intensity of the similarly sized clusters and plotted on a relative scale. The dashed lines are guides to the eye and demonstrate the different behavior of a constantly growing average charge state versus the rapid saturation of the dominant charge state. The ion charge states are estimated without considering the initial ion kinetic energy release.

state (represented by open circles Fig. 3) is determined as the center of gravity of the ion yield spectra. It rises monotonically from $q \approx 5^{+}$to $q \approx 15^{+}$within the investigated power-density window, indicating an increase in overall energy absorption by the cluster with increasing power densities. The consequences are a higher mean charge state and higher kinetic energy releases, which both contribute to the observed slope. The dominant charge state (represented by black diamonds in Fig. 3) is defined as the most abundant one in the ion yield spectra. It rises rapidly and saturates just above $26^{+}$. It should be noted that the charge states are deduced from the spectra without considering initial kinetic energy releases which push the ion peaks to shorter flight times. The observed saturation points toward similar photoionization processes for xenon atoms in the gas phase and in the cluster. $Q=26^{+}$is also the highest observed charge state in the atomic xenon spectra (cf. Fig. 2). The ground state ionization potential for $\mathrm{Xe}^{25+}$ is with $890 \mathrm{eV}$ [25] slightly higher than the incident photon energy of $800 \mathrm{eV}$, indicating that sequential multiphoton ionization via resonant excitation and intermediate excited states can occur [26], similar to observations in argon atoms and clusters [6].

The absence of low charge states and the rapid increase of the dominant $q$ with increasing power densities points to a very efficient suppression of recombination processes in the X-ray induced nanoplasma. It is noted that this observation is quite surprising, as for the most highly charged clusters less than $1 \%$ of the photoactivated electrons can leave before the photoemission is frustrated in the 
increasing cluster Coulomb potential [13]. Our findings are also vastly different as compared to results of earlier experiments with about one order of magnitude lower photon energies where very efficient nanoplasma recombination mechanisms have been found $[17,19]$. The initial photoionization processes are similar for both cases, and they are guided by the atomic photoabsorption cross sections and subsequent inner-shell vacancy decay. After frustration of the photoemission, a nanoplasma is formed in which the electrons thermalize within femtoseconds through energy-exchanging collisions $[7,15]$. The present results show that the high x-ray photon energies, and thus high electron excess energies and mean charge states, must play an important role for the nanoplasma evolution.

For a further discussion of the nanoplasma dynamics, we have implemented a simplified model of the cluster expansion in the time-dependent plasma code FLYCHK [27]. FLYCHK is a population kinetics model that includes all the relevant ionization and recombination processes for arbitrary atomic elements. The disintegration of large clusters can be approximated by a hydrodynamic expansion in which the hot quasifree electrons force the cold ions to expand. FLYCHK is then used to calculate the ionization and population distribution in the expanding nanoplasma up to several nanoseconds after the x-ray pulse. As the microscopic ionization dynamics cannot be described with FLYCHK, two different initial states in qualitative agreement with the experimental data shown in Fig. 2 are assumed. In the first scenario representing the high $\mathrm{x}$-ray power density case, a quasiequilibrium plasma with a mean charge state around the highest observed $q=26^{+}$and solid Xe density is taken as the starting point. To represent the conditions from the regions with about an order of magnitude less power density, a colder nanoplasma with a mean charge state of $8^{+}$is assumed. The results of the FLYCHK calculations and the corresponding experimental results from Figs. 2(a) and 2(b) are displayed in Fig. 4. In comparison with the experiment, FLYCHK reproduces qualitatively the measured ion distribution of case (a) including the suppressed recombination to lower charge states but predicts too much recombination for case (b). The simulations show that for the hot, fast expanding plasma in case (a) the high initial electron temperature lowers three-body recombination rates during the time scale where the plasma is still dense and that the high charge states freeze out whereas in (b) the initial lower electron temperatures lead to a slower expansion and therefore a longer period of efficient recombination. It should be noted that our model assumes homogeneous plasma densities thus neglecting any shell effects [17] and starts at quasiequilibrium conditions. Charge redistribution within the nanoplasma and shell explosion can lead to a significant broadening of the ion distribution and higher observed charge states [18]. Further, FLYCHK does not take into account three-body recombination into higher Rydberg states above $n=10$,

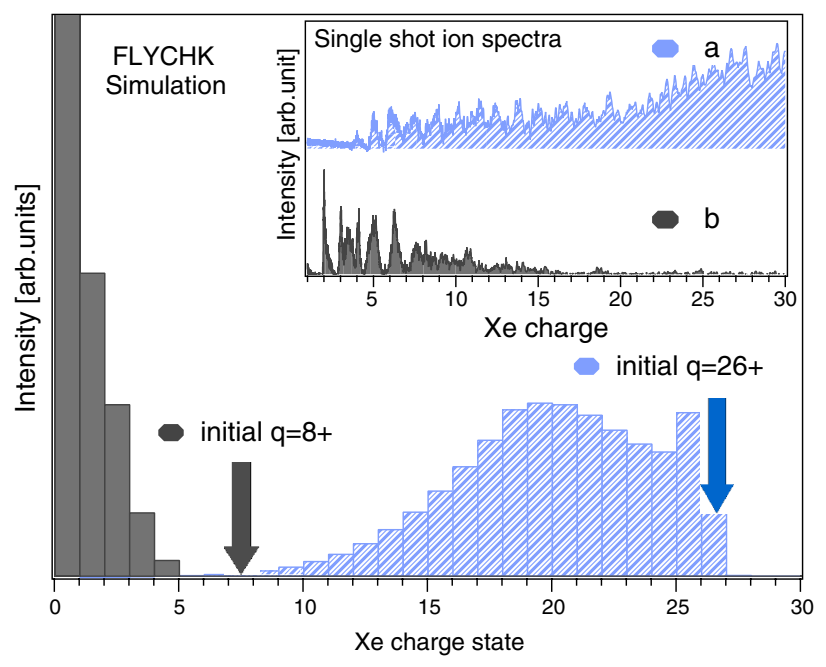

FIG. 4 (color online). Comparison between FLYCHK simulations and experimental single-shot ion data (inset). Both the data and simulation show a strong suppression of recombination for the highly excited x-ray-induced nanoplasma (initial $q=26^{+}$) in the center of the FEL focus and a significant recombination for lower excitation (initial $q=8^{+}$). For the initial $q=26^{+}$nanoplasma, the high charge states freeze out due to rapid expansion of the cluster and low three-body recombination rates for hot electron temperatures.

which have been shown to become significant for clusters explosions induced with intense infrared pulses after several ps of expansion [28].

In summary, thanks to the single-shot coincidence method pioneered in this work, the $\mathrm{x}$-ray induced ionization dynamics of Xe clusters could be studied under unprecedented well-controlled experimental conditions, i.e., at well-defined power density and for well-characterized particle size. This way, the strong limitation of integrating over very different power densities and cluster sizes in the focal volume common to almost all studies of nonlinear light-matter interaction could be overcome. At the highest power density of $\left(5 \times 10^{16} \mathrm{~W} / \mathrm{cm}^{2}\right)$, predominantly ions with charge states peaking at $26^{+}$are observed while low charge states are virtually absent. The results give strong evidence that the narrow charge distribution is due to inefficient electron-ion recombination. The inefficient recombination is attributed to the high initial temperature of the $\mathrm{x}$-ray produced nanoplasma.

This research was carried out at the Linac Coherent Light Source (LCLS) at the SLAC National Accelerator Laboratory. LCLS is an Office of Science User Facility operated for the U.S. Department of Energy Office of Science by Stanford University. The authors want to thank Y. Ralchenko and R. W. Lee for their support with the FLYCHK simulations as well as T. Fennel, J.-M. Rost, and U. Saalmann for helpful discussions. We acknowledge the Max Planck Society for funding the development and operation of the CAMP instrument within the ASG at 
CFEL and funding from BMBF 05KS4KT1, 05KS7KT2 and 05K10KT2, DFG BO 3169/2-2, as well as HGF Virtuelles Institut VH-VI-302.

*Corresponding author. bostedt@slac.stanford.edu

[1] H. N. Chapman et al., Nature (London) 470, 73 (2011).

[2] M. M. Seibert et al., Nature (London) 470, 78 (2011).

[3] L. Young et al., Nature (London) 466, 56 (2010).

[4] M. Hoener et al., Phys. Rev. Lett. 104, 253002 (2010).

[5] H. Thomas et al., Phys. Rev. Lett. 108, 133401 (2012).

[6] S. Schorb, D. Rupp, M. L. Swiggers, R. N. Coffee, M. Messerschmidt, G. Williams, J. D. Bozek, S.-I. Wada, O. Kornilov, T. Möller, and C. Bostedt, Phys. Rev. Lett. 108, 233401 (2012).

[7] S. M. Vinko et al., Nature (London) 482, 59 (2012).

[8] A. Barty et al., Nature Photon. 6, 35 (2012).

[9] L. Lomb et al., Phys. Rev. B 84, 214111 (2011).

[10] T. Ditmire, T. Donnelly, A. M. Rubenchik, R. W. Falcone, and M. D. Perry, Phys. Rev. A 53, 3379 (1996).

[11] T. Fennel, K.-H. Meiwes-Broer, J. Tiggesbäumker, P.-G. Reinhard, P. M. Dinh, and E. Suraud, Rev. Mod. Phys. 82, 1793 (2010).

[12] H. Wabnitz et al., Nature (London) 420, 482 (2002).

[13] C. Bostedt et al., Phys. Rev. Lett. 100, 133401 (2008).

[14] H. Iwayama et al., J. Phys. B 42, 134019 (2009).
[15] C. Bostedt, H. Thomas, M. Hoener, T. Möller, U. Saalmann, I. Georgescu, C. Gnodtke, and J.-M. Rost, New J. Phys. 12, 083004 (2010).

[16] C. Bostedt et al., Phys. Rev. Lett. 108, 093401 (2012).

[17] M. Hoener, C. Bostedt, H. Thomas, L. Landt, E. Eremina, H. Wabnitz, T. Laarmann, R. Treusch, A. de Castro, and T. Möller, J. Phys. B 41, 181001 (2008).

[18] H. Thomas, C. Bostedt, M. Hoener, E. Eremina, H. Wabnitz, T. Laarmann, E. Plönjes, R. Treusch, A. R. B. De Castro, and T. Möller, J. Phys. B 42, 134018 (2009).

[19] B. Ziaja, H. N. Chapman, R. Santra, T. Laarmann, E. Weckert, C. Bostedt, and T. Möller, Phys. Rev. A 84, 033201 (2011).

[20] L. Strüder et al., Nucl. Instrum. Methods Phys. Res., Sect. A 614, 483 (2010).

[21] J. D. Bozek, Eur. Phys. J. Special Topics 169, 129 (2009).

[22] S. Schorb et al., Appl. Phys. Lett. 100, 121107 (2012).

[23] M. Hoener, C. Bostedt, S. Schorb, H. Thomas, L. Foucar, O. Jagutzki, H. Schmidt-Boeking, R. Dörner, and T. Möller, Phys. Rev. A 78, 021201 (2008).

[24] C. Bostedt, M. Adolph, E. Eremina, M. Hoener, D. Rupp, S. Schorb, H. Thomas, A. R. B. De Castro, and T. Möller, J. Phys. B 43, 194011 (2010).

[25] T. A. Carlson, C. W. Nestor, Jr., N. Wasserman, and J. D. Mcdowell, At. Data Nucl. Data Tables 2, 63 (1970).

[26] B. Rudek et al. (to be published).

[27] H.-K. Chung, M. Chen, W. Morgan, Y. Ralchenko, and R. Lee, High Energy Density Phys. 1, 3 (2005).

[28] T. Fennel, L. Ramunno, and T. Brabec, Phys. Rev. Lett. 99, 233401 (2007). 